\title{
UNA REINTERPRETACIÓN DE LA VIOLENCIA EN LAS ESCUELAS
}

\section{Uma reinterpretação da violência nas escolas}

\author{
Maria Jesús Comellas Carbó
}

D ra en Psicología. Profesora de la UAB, Coordinadora del grupo GRODE, D irectora delO bservatorio delaviolencia en las escuelas- Espanha, e-mail:Mjcomellas@ grode.org

\section{Resumen}

Los hechos de violencia en los centros escolares llaman la atención por su inadecuación y gravedad y son fruto de procesos que no siempre tienen una respuesta adecuada y de unas circunstancias que, en un momento específico, los posibilitan. Según sea el tratamiento del tema puede generar alarmismo entre iguales, entre el profesorado y las familias, y en la misma sociedad que realimenta el debate y dificultar la aplicación de respuestas y acciones educativas para reducirla y prevenirla. Es preciso hacer, sin más dilación, un análisis en profundidad tanto de lo que pasa, por qué se producen estas reacciones, la forma de interpretarse y, especialmente, las alternativas educativas a implementar. El interés del grupo G RODE (Grup de Recerca, O rientació i D esenvolupament Educatiu) por estos fenómenos de violencia, parte del estudio de este fenómeno y del análisis compartido con el profesorado sobre las formas y procesos de socialización y el aprendizaje relacional del alumnado en los centros educativos, abriendo la mirada hacia el entorno (actividades extraescolares, grupos culturales, deportivos...). Se presenta el enfoque

Rev. Diálogo Educ., Curitiba, v. 9, n. 28, p. 417-439, set./ dez. 2009 
educativo que da lugar a la investigación que se lleva a cabo en centros escolares, públicos y concertados, de Educación Primaria y Secundaria de Catalunya. La metodología seguida se basa en el modelo de Investigación acción participativa con el profesorado. Los agentes profesionales próximos al centro educativo, como servicios educativos (psicopedagogía, recursos) y trabajo social, entre otros, también participan aportando su visión y su experiencia.

Palabras-clave: Fenómenos de violencia. Procesos de socialización. A prendizaje relacional. Centros educativos.

\section{Resumo}

Os atos de violência nas escolas chamam a atenção por sua inadequação e gravidade, e são o fruto de processos que nem sempre têm uma resposta adequada e de circunstâncias que, em um momento específico, os possibilitam. A forma de tratamento dado ao tema pode provocar alarmismo entre alunos, professores, famílias e mesmo na sociedade, o que realimenta o debate e dificulta a implementação de respostas e ações educativas para reduzi-lo e preveni-lo. É preciso fazer, sem mais delongas, uma análise profunda do que ocorre, porque essas reações são produzidas, as formas de interpretá-las e, especialmente, as alternativas educativas a serem implementadas. O interesse do grupo GRODE (Grupo de Recerca, Orientació i Desenvolupament Educatiu) pelos fenômenos de violência, parte do estudo deste fenômeno e da análise compartilhada com os professores sobre as formas e processos de socialização e a aprendizagem relacional dos alunos nas escolas, abrangendo no olhar o entorno das escolas (atividades extraescolares, grupos culturais, desportivos). No artigo se apresenta o enfoque educativo que sustenta a investigação realizada em escolas públicas e privadas de educação primária e secundária da Catalunha. A metodologia seguida é baseada no modelo da pesquisa-ação participativa com os professores. Os agentes profissionais próximos à escola, que prestam serviços educativos (psicopedagogia, recursos) e trabalho social, entre outros, também participam, contribuindo com sua visão e experiências.

Palavras-chave: Fenômeno de violências. Processos de socialização. Aprendizagem relacional. Escolas.

Rev. Diálogo Educ., Curitiba, v. 9, n. 28, p. 417-439, set./ dez. 2009 


\section{INTRODUCCIÓN}

Las informaciones actuales, en relación a las dificultades de convivencia en los centros escolares, acoso entre iguales, violencia, rechazo a situaciones y características personales (diversidad en todas sus vertientes), exigen, sin más dilación, un análisis en profundidad tanto de lo que pasa, por qué se producen estas reacciones, la forma de interpretarse y las alternativas educativas a implementar.

Para poder hacer este análisis es preciso un punto de partida común en relación al mismo concepto de violencia ya que su significado es polisémico y varía en función del campo de conocimiento que trata el tema y de la perspectiva teórica desde la cual se aborda. Las disciplinas más comunes que lo analizan son la Antropología, la Sociología, la Psicología, las Ciencias Políticas, las Ciencias de la Educación y las Ciencias de la Comunicación y el mundo jurídico.

Como sucede con los términos "normal" y "patológico", su definición no es neutral y menos todavía cuando, a partir de su detección y evaluación, se deben tomar medidas de control que garanticen la seguridad de las personas.

La violencia parte de una situación de interacción, cuando uno 0 varios actores participan de forma directa o indirecta, masivamente 0 de forma aislada, para vulnerar las pertenencias de una o varías personas o herirlas en algún aspecto personal o de su contexto (ROJAS, 2005).

En el ámbito escolar, a manera explicativa, se dan algunas manifestaciones violentas que pueden relacionarse, entre las principales que subyacen en estas reacciones, con una dificultad en el establecimiento de los vínculos, una excesiva presión social sobre las personas, la influencia de ciertos modelos, miedo a perder la preponderancia en el grupo, respuesta ante tensiones y rechazos recibidos o una demanda de atención ante la exclusión.

Se debe considerar el ambiente, el rol de las personas adultas y las dificultades adaptativas que puede tener el alumnado (HAMON, 2004) como factores contextuales ya que, en muchos momentos, se genera una presión difícil de gestionar y que puede manifestarse con episo dios de violencia en las relaciones poco adecuadas entre los iguales, con las personas adultas o el seno del mismo contexto escolar.

Estas manifestaciones de violencia, cuantitativamente minoritarias, llaman la atención por su gravedad (MABIO N-BO NFILS, 2005) pero son fruto de procesos educativos y sociales, circunstancias personales y especialmente se dan en el contexto de la interacción por

Rev. Diálogo Educ., Curitiba, v. 9, n. 28, p. 417-439, set./ dez. 2009 
lo que no pueden analizarse sólo mirando las personas actoras (quien hace y quien recibe) sino estudiando los comportamientos, tengan el signo que tengan, dentro del contexto sistémico dónde tienen lugar y donde se les da significado.

Según este enfoque, la violencia y sus manifestaciones son un fenómeno complejo que se inscribe en las cadenas de interacciones (visibles o no) entre las personas en nuestro caso dentro del contexto escolar. Las causas deben analizarse desde un enfoque circular que explora los acontecimientos, el entorno dónde se dan, los diferentes elementos o factores asociados que interactúan entre ellos, las personas, los mecanismos de regulación y sus resultados.

Esta mirada se contrapone al método lineal a partir del que se analizan los problemas según un esquema de causa - efecto y se buscan soluciones simples einmediatas a los problemas presentados (MEIRIEU, 2001). Las soluciones son complejas puesto que unos comportamientos similares pueden ser originados por causas variadas y de igual manera unas causas similares pueden tener unos resultados muy diferentes.

Por todo esto queremos dar relieve a la afirmación de que no hay, en edad escolar, un perfil de personas susceptible de actuar según patrones predeterminados, sino que potencialmente la totalidad de la población se puede encontrar involucrada en todo tipo de acciones en un momento $\mathrm{u}$ otro. Como consecuencia se debe actuar en el marco de los centros educativos para llegar a la totalidad de la población, a lo largo de toda la escolaridad, con la participación del profesorado y el apoyo de las familias y servicios educativos del territorio (CO MELLAS; LOJO , 2008).

La convivencia sólo puede tratarse con toda su complejidad y riqueza y no únicamente en situaciones puntuales. Se deben incluir todos los lugares dónde se construye el proceso de socialización y aprendizaje de la convivencia con una perspectiva global. Por esto la responsabilidad no debe recaer exclusivamente en el profesorado, sino que es necesaria la implicación de toda la comunidad educativa (RAVEAUD, 2006).

Lógicamente las problemáticas emergen dentro del centro educativo, puesto que allí convive toda la población infantil y adolescente durante muchas horas al día. En el aula, espacio dónde se realiza la actividad más regulada, y en los otros espacios escolares (pasillos, patio... ), es dónde aparecen tensiones y dificultades, y es dónde se debe llevar a cabo la acción educativa.

En el momento en que se hacen actuaciones paliativas si afrontan el tema de forma individual quedarán sin valorarse los factores que han provocado la situación. Estas actuaciones pese a tener una eficacia a corto

Rev. Diálogo Educ., Curitiba, v. 9, n. 28, p. 417-439, set./ dez. 2009 
plazo corren el riesgo de no tratar las causas con lo que se acaba fragilizando más la población ya de por sí vulnerable. Se debe, pues, superar este enfoque individual para pasar a una perspectiva global del grupo y disponer de una mirada multiprofesional (MARCHE SI, 2005).

\section{El aprendizaje relacional de la infancia a la adolescencia: familia y escuela}

La acción educativa y el aprendizaje de la socialización y maduración se lleva a cabo en el contexto donde se establecen las relaciones en un proceso dialéctico con el entorno, con todos sus agentes y sobre todo con una participación clara de cada grupo de adultos que lo acompañan. El objetivo principal es llegar a comprender la propia identidad, crecer y aprender las condiciones de las relaciones interpersonales y aprender las habilidades para poder actuar.

Los primeros vínculos afectivos que dan pie a la construcción de la propia identidad: A utoimagen, se crean en la familia. La aceptación de la individualidad, las respuestas que se reciben ante las demandas vitales y la apertura al mundo social serán los ejes que abrirán la puerta al proceso de socialización.

Muy pronto las relaciones con el grupo familiar amplio: fratria, familia extensa, vecindario... abrirán un universo complejo en el que las expresiones emocionales, tensiones y deseos van a recibir respuestas diferenciales y añadirán riqueza a este modelado social.

La comparación y contraste entre la imagen de la propia familia y la de los demás será un factor clave de reconstrucción de la identidad y paso fundamental para poder acceder a un proceso de socialización más amplio y adquirir seguridad y autonomía para poder tener una mirada más objetiva que de paso a la autoestima y autoconcepto (BRUNER, 1998).

Es también en el núcleo familiar dónde se aprende las diferentes formas de comunicación: verbal y no verbal. Los códigos, los significados, la manera de dirigirse a los iguales, a los adultos conocidos o a los extraños configuran el bagaje para comunicarse en contextos diferentes y poder expresar de forma estructurada ideas, pensamientos y sentimientos.

Los mismos rituales no tienen el mismo significado según las comunidades. Igualmente las palabras o gestos, cambian de significado según quien los dice, dónde y cómo, hecho que comporta una gran complejidad y, en algunos casos, dificultades de comprensión y problemas relacionales.

Rev. Diálogo Educ., Curitiba, v. 9, n. 28, p. 417-439, set./ dez. 2009 
Con la entrada en la institución educativa se pasa a una interacción con un gran repertorio de modelos y conductas que entrarán en contraste con las experiencias personales y los modelos adquirido hasta este momento $\mathrm{y}$, a lo largo de la escolaridad, se deberá guiar, el nuevo aprendizaje que ha de permitir posicionarse para seleccionar, imitar, rechazar o compartir valores, formas, actitudes y conductas (CO MELLAS, 2004).

\section{La dinámica relacional y las dificultades de convivencia}

Este proceso de socialización en el centro escolar debefavorecer el desarrollo integral de las personas partiendo del reconocimiento que se merecen como tales. De esta manera todo el mundo, todo el alumnado, debe tener un espacio relacional positivo y la posibilidad de valoración de sus competencias, potencial, calidades y necesidades.

Por esto la educación debe llegar a la totalidad de la población y el reto educativo de la institución escolar se centra en la construcción de un marco pedagógico que incluya a todo el mundo para favorecer la convivencia, respeto y consideración entre el alumnado, entre las personas adultas que educan y entre adultos y alumnado (MEIRIEU, 2004).

Es en el aulay en la escuela donde se manifiestan, con más fuerza, los diferentes modelos educativos recibidos previamente, los efectos de las múltiples experiencias personales y el resultado de las influencias exteriores que condicionan actitudes, intereses y motivaciones ante el hecho escolar (PUGEAULT-CICCHELLI; CICCHELLI; RAG I, 2004).

En el grupo se producen interacciones, exploran conductas, incorporan actitudes y valores, afectos y desafectos, conflictos con resolución o no, y todo pasa de forma explicita o no (currículum oculto).

En el grupo se actúa o se reacciona, se explicitan o no expectativas y se construyen atribuciones y prejuicios. El lenguaje corporal, actitudinal, alianzas, roles, liderazgos y rechazos se van forjando con el tiempo y es complicado el cambio de manera espontánea. Por esto pesa tanto la imagen que el grupo da a cada persona puesto que acabará interiorizándose como autoimagen.

Las experiencias que el alumnado tenga dentro los grupos dónde crece influirán en su desarrollo individual y social, tanto en positivo como en negativo. Por esto uno de los ejes en los que es preciso incidir es en la dinámica, las relaciones y la cohesión del grupo.

Los vínculos para ser positivos no precisan tener la misma intensidad pero sí que deben posibilitar las relaciones grupales puesto

Rev. Diálogo Educ., Curitiba, v. 9, n. 28, p. 417-439, set./ dez. 2009 
que son la base para que el alumnado construya su imagen social que ha de dar pie a su desarrollo psicoemocional. El aula como contexto privilegiado permite al profesorado reflexionar sobre las relaciones, ver los valores inherentes, las representaciones que puede tener cada persona del grupo e intervenir para resolver determinados conflictos que, en definitiva, afectan a cada persona del grupo (VAN HAECHK, 2001), unas porque son víctimas y otras porque lo gestionan o lo observan, y practicar competencias sociales que favorecen una convivencia democrática (SANTO S G UERRA, 2002).

En todos los casos el lenguaje utilizado, por el profesorado o el propio alumnado, para relatar los hechos, justificar algunos comportamientos o reacciones, favorece la construcción de la realidad, da fortaleza a la percepción de las relaciones y condiciona (contrarresta o refuerza) prejuicios, atribuye causas, construye expectativas que influirán en las miradas, interpretaciones y acciones posteriores (CO MELLAS, 2002).

Cuanto mayor sea el consenso sobre los descriptores con que se definen las situaciones (personas, hechos, acontecimientos), mayor será la dificultad para cambiar los prejuicios, reducir o anular su efecto y mayor será el riesgo de que las personas objeto de la descripción acaben comportándose de forma que se refuerce la visión que de ellas se tiene (profecías auto cumplidoras).

El objetivo educativo fundamental al que el profesorado debe responder es, pues, atender las redes de comunicación que se establecen dentro el grupo, identificar los liderazgos y sus estilos, las causas de su creación y mantenimiento, conocer la coherencia entre la posición real y la percibida por cada miembro dentro el grupo y será en este marco grupal, donde deberállevarse a cabo la intervención educativa(GABERAN, 2005).

Por ello deberá ejercer su liderazgo para incidir en el clima, potenciar las oportunidades educativas, favorecer la convivencia y crear un espacio de pertenencia para todo el alumnado con un debate positivo ante las dificultades.

\section{Los factores de vulnerabilidad relacional}

Cuando hablamos de vulnerabilidad hacemos referencia a las dificultades que afectan a una persona o grupo para poder tener unas relaciones positivas.. Esta vulnerabilidad, a menudo atribuida a quien tiene estas dificultades, puede estar determinada por múltiples factores

Rev. Diálogo Educ., Curitiba, v. 9, n. 28, p. 417-439, set./ dez. 2009 
que provocan que el grupo no construya un espacio necesario para poder relacionarse positivamente.

La mayoría de estos factores de vulnerabilidad, cuando son individuales, están vinculados a estereotipos sociales predeterminados con la categoría de "diversidad o anormal". Este hecho comporta la creación paralela de. "modelos NO deseables".

Estos atributos pueden alejar o aislar a una persona de su grupo lo que provoca situaciones dolorosas y excluyentes. Entonces esta persona puede serpercibidapor el grupo como más débil y vulnerabley puede serfoco de rechazo o marginación. Ella ante esta situación puede actuar inhibiéndose, alejándose 0 actuando de forma inadecuada (violencia, indisciplina, rechazo a la dinámica del grupo... ) (D UBET FRANÇO IS, 2004).

La detección y análisis en profundidad de estos factores de vulnerabilidad, aportará datos para encontrar posibles indicadores explicativos (no justificativos) que interfieren y dificultan el proceso de socialización y convivencia y que pueden condicionar unas acciones grupales y lógicamente individuales.

Entre estos factores podemos encontrar el bajo rendimiento escolar, imagen corporal (malformación, obesidad, rasgos físicos) aspectos culturales, recursos socioeconómicos, déficit o trastornos más o menos relevantes, intereses de juego, actividades o elección personal de estilos de vida.

Con esto se constata que el problema relacional no lo tiene una persona sino el grupo puesto que los motivos de la exclusión dependen de los significados e interpretaciones que hacen las personas del grupo de cada uno de sus miembros. Q uienes lideran el grupo, lo contempla, lo reciben son personas implicadas en la dinámica y es en esta red relacional donde se ha de intervenir.

Con la dinámica relacional las personas construyen las percepciones del lugar que ocupan en el grupo y el grado de protagonismo de cada miembro. El sentimiento de pertenencia, de vulnerabilidad 0 exclusión serán determinantes de actitudes y respuestas individuales y grupales ante los requerimientos del grupo y del profesorado y es donde se ha de intervenir a partir del análisis (MUCHIELI, 2002).

D e esta manera se puede evitar la focalización de las reacciones en la individualidad. Una actitud grupal positiva evitaría justificar situaciones de exclusión: "se lo ha buscado", "no tiene habilidades relacionales", "tienen una sensibilidad demasiada acusada" frases que se atribuyen a las personas marginadas 0 excluidas

Con el análisis de estos factores el profesorado descubre las reglas relacionales del grupo factor clave para tomar decisiones sobre

Rev. Diálogo Educ., Curitiba, v. 9, n. 28, p. 417-439, set./ dez. 2009 
cómo se ha de intervenir, en el marco grupal, para favorecer la construcción de pautas de convivenciamás inclusivas, prevenir situaciones de marginación y no focalizar las respuestas sólo en el ámbito individual (TOCZEK; MARTINOT, 2004).

\section{El profesorado y profesionales favor clave de la convivencia}

Se trata, pues, de cambiar la mirada dentro el sistema relacional y de socialización. El protagonismo y rol de la institución educativa de referencia: la escuela, es clave para fortalecer la co hesión social V an Haechk, (2006) y el entendimiento entre los pueblos, entre familia y escuela y con los agentes del contexto social en el que se vive (MORIN, 2007).

Los reproches que familia y escuela se hacen mutuamente o que la sociedad hace a ambos influencian en gran manera en las actitudes de los menores durante este complejo proceso de aprendizaje de la convivencia.

Por esto y teniendo presente la dificultad de este aprendizaje: códigos, habilidades, competencias hace falta que se considere la convivencia como objetivo común y un aprendizaje fundamental en el marco escolar y de la comunidad.

\section{El proyecto que se propone}

El proyecto que se presenta en el marco del $\mathbf{0 b s e r v a t o r i o ~ d e ~ l a ~}$ violencia a las escuelas parte de la base de un análisis sistemático en la que el centro educativo es el núcleo del sistema relacional del alumnado por disponer de toda la población y por ser quien representa a la sociedad y responsable de llevar a cabo el proceso educativo para toda la población como un derecho inapelable (CO MELLA S-LOJO, 2006).

El modelo en el que se basa el trabajo es el de: Investigación acción participativa con el profesorado y los agentes profesionales próximos al centro educativo: trabajo social, mediación socio comunitaria, responsables de proyectos de infancia y adolescencia, servicios municipales y de la comunidad. Sus objetivos son:

- analizar, con los colectivos profesionales los conceptos, las dinámicas que se dan en los grupos de aula para poder identificar factores de riesgo en relación al proceso de

Rev. Diálogo Educ., Curitiba, v. 9, n. 28, p. 417-439, set./ dez. 2009 
socialización, posibles dinámicas y cohesión grupal que pueden abocar a situaciones de violencia en la escuela;

- compartir y comprender, con los diferentes profesionales y agentes de la comunidad educativa, las señales y los síntomas que pueden interferir en la dinámica del aula;

- debatir las prácticas y actuaciones encaminadas a dar respuestaalas situaciones educativasy las respuestas puntales, en el momento en que se den;

- generar estrategias para optimizar la intervención desde una perspectiva educativa, inclusiva y sistémica como factor potenciador de la socialización y prevención de la violencia.

Lapoblación objeto denuestro trabajo se distribuye en diferentes perfiles según el momento del proceso: profesionales / alumnado.

Profesionales, en este colectivo destacamos:

Profesorado del centro educativo: participan del proceso 101 centros educativos entre los que hay diferentes perfiles: Concertados, Públicos de Primaria y secundaria (Tabla anexo 1). En cada centro se busca la implicación del equipo directivo, no siempre posible, como responsable y facilitador de los pasos del proceso para incorporar los acuerdos y análisis que se propongan. Es importante la participación del profesorado que tiene la tutoría en el aula. El número de participantes es variable en función de las posibilidades de cada centro.

Profesionales de la comunidad: las personas de la comunidad que participan en las sesiones de análisis y debate son diferentes según la zona en la que se lleva a cabo y los recursos de que se dispone. El perfil es: Equipo de asesoramiento psicopedagógico, Agentes educativos que trabajan para la cohesión social, Mediación e integración socio comunitaria.

Alumnado: grupos de primaria, a partir de $3 r$ curso de primaria (8-12 años) e grupos de secundaria, especialmente en la secundaria obligatoria (12-16).

Rev. Diálogo Educ., Curitiba, v. 9, n. 28, p. 417-439, set./ dez. 2009 
TABLA 1 - Titularidad del Centro y Sexo

\begin{tabular}{cccccc}
\hline $\begin{array}{c}\text { Titularidad } \\
\text { del Centro }\end{array}$ & \multicolumn{2}{|c|}{ Sexo } & \multicolumn{2}{c}{ Total } \\
\hline Pública & 3498 & 3522 & 7020 & 80,84 \\
Concertada & 777 & 892 & 1669 & 19,16 \\
\hline Total № & 4275 & $\begin{array}{c}4414 \\
\text { \% }\end{array}$ & $\begin{array}{c}89,20 \\
50,0\end{array}$ & 100 & 100 \\
\hline
\end{tabular}

Fonte: Campo de pesquisa, organizado pela autora.

El número de alumnado y los grupos lo decide el claustro, según las personas, los proyectos que ya realizan en el centro o la valoración que hacen del proyecto.

En ningún caso se lleva a cabo el trabajo en un solo curso de un nivel determinado (que es donde pueden haber más dificultades relaciónales) sino en todos los grupos del nivel de edad elegido.

\section{El proceso sigue las siguientes fases}

1) planteamiento a los equipos de los centros y de la comunidad de los objetivos del proyecto;

2) información en relación al proceso y enfoque centrado en la dinámica del aula.

3) realización del sociograma, por parte del alumnado, con la aplicación del software creado para este fin acompañados por el profesorado del aula;

4) selección del alumnado considerado de interés por su posible protagonismo o vulnerabilidad en función de la relevancia de datos considerados como significativos: Liderazgo, Exclusión, Expansividad Positivay Negativa, Autovaloración desajustada;

5) aportación de Información pedagógica del alumnado por el profesorado, y otros agentes conocedores de los sujetos de la muestra;

Rev. Diálogo Educ., Curitiba, v. 9, n. 28, p. 417-439, set./ dez. 2009 
6) dD ebate compartido para contrastar estas informaciones y comprender las causas de dicha vulnerabilidad;

7) toma de decisiones en relación a las actuaciones que el profesorado realizará, SIEMPRE EN EL SENO DEL GRUPO , encaminadas aincidiren las causas de vulnerabilidad y mejorar las relaciones grupales.

\section{Instrumentos}

$\rightarrow$ Software aportado por el grup GRO DE: http:/ / www.portal.grode.org que permite una aplicación y recogida de informaciones de forma rápida y precisa del sociograma desde el punto de vista cuantitativo y gráfico;

$\rightarrow$ breve escala de indicadores pedagógicos para sistematizar la información del profesorado;

$\rightarrow$ informaciones cualitativas que aporta el profesorado como agente observador directo de la dinámica y relaciones en el centro educativo.

\section{RESULTADOS}

\section{Debate inicial}

En las sesiones posteriores sellevan a cabo desde dos modalidades:

$\rightarrow$ D ebate entre centros

$\rightarrow$ D ebate en claustro de cada centro (en experimentación)

Debate entre centros: los encuentros que se llevan a cabo con la participación de diferentes centros educativos, siguen el proceso siguiente:

a) la interpretación de los datos;

- causa de las relaciones;

- reacción ante la mirada, ambiente o comportamiento grupales;

Rev. Diálogo Educ., Curitiba, v. 9, n. 28, p. 417-439, set./ dez. 2009 
- las causas individuales;

- las dinámicas de aula o centro;

- las respuestas del profesorado.

b) análisis de las acciones o factores que pueden interferir en la mejora de la convivencia y pueden reforzar algunas respuestas negativas;

c) acciones que se llevan a cabo en el centro educativo o en el entorno como elementos favorecedores de la convivencia: dificultades, oportunidades y formas de compartirse;

d) el debate colectivo necesario en este tema;

e) acciones y cambios que se pueden generar a partir de estas informaciones (experiencias compartidas, dificultades y formas de resolverlas);

- debates;

- aspectos organizativos;

- aspectos didácticos;

- proyectos compartidos con otros centros;

- formación del profesorado;

- implicación de profesionales vinculados con le mejora del sistema educativo;

- implicación institucional.

Debate en claustro de cada centro (en experimentación): por el interés en el seguimiento y mejorar la participación se ha propuesto hacen encuentros en cada centro, este curso se iniciará con carácter experimental.

\section{Análisis provisional de los aspectos más relevantes: en zonas sombreadas se han marcado los datos de mayor interés.}

a) Liderazgo ( $n^{0}$ de elecciones recibidas) Personas con ascendente en el grupo y cuyas actitudes pueden tener

Rev. Diálogo Educ., Curitiba, v. 9, n. 28, p. 417-439, set./ dez. 2009 
un efecto amplificador de formas de relacionarse. Pueden ser un referente de lo que se valoriza en el grupo. Se constata que 330 alumnas y 431 alumnos (761 en total) no han tenido elecciones 0 sólo 1 , lo que representa una gran vulnerabilidad por los pocos o nulos vínculos relacionales con el grupo.

TABLA 2 - Elecciones Recibidas* Sexo

\begin{tabular}{|c|c|c|c|c|c|}
\hline & & \multicolumn{2}{|c|}{ Sexo } & \multirow{2}{*}{$\begin{array}{l}\text { Total } \\
\text { Chica }\end{array}$} & \multirow[b]{2}{*}{$\%$} \\
\hline & & Chica & Chico & & \\
\hline \multirow{6}{*}{$\begin{array}{l}\text { Liderazgo } \\
\text { Elecciones } \\
\text { Recibidas }\end{array}$} & Oo 1 Eleociones & 330 & 431 & 761 & 8,75 \\
\hline & $\begin{array}{l}\text { Entre 2-5 } \\
\text { Elecciones Recibidas }\end{array}$ & 1369 & 1488 & 2857 & 32,94 \\
\hline & $\begin{array}{l}\text { Entre } 6-9 \\
\text { Elecciones Recibidas }\end{array}$ & 1272 & 1234 & 2506 & 28,83 \\
\hline & $\begin{array}{l}\text { Entre 10-13 } \\
\text { Elecciones Recibidas }\end{array}$ & 717 & 665 & 1382 & 15,90 \\
\hline & $\begin{array}{l}\text { Entre 14-17 } \\
\text { Elecciones Recibidas }\end{array}$ & 253 & 253 & 506 & 5,81 \\
\hline & $\begin{array}{l}\text { Mas de } 17 \\
\text { Elecciones Recibidas }\end{array}$ & 334 & 343 & 677 & 7,78 \\
\hline Total & & 4275 & 4414 & 8689 & \\
\hline
\end{tabular}

Fonte: Campo de pesquisa, organizado pela autora. del grupo.

El 2858 personas tienen menos del 20\% de relaciones positivas

Rev. Diálogo Educ., Curitiba, v. 9, n. 28, p. 417-439, set./ dez. 2009 
TABLA 3 - Índice de Popularidad * Sexo

\begin{tabular}{ll|cc|c}
\hline & & \multicolumn{2}{|c|}{ Sexo } & Total \\
\cline { 3 - 5 } & & Chica & Chico & Chica \\
\hline $\begin{array}{l}\text { Índice de } \\
\text { Popularidad }\end{array}$ & $\begin{array}{l}\text { Bajo entre 0i 20\% } \\
\text { de Elecciones }\end{array}$ & $\mathbf{1 3 3 5}$ & $\mathbf{1 5 2 3}$ & $\mathbf{2 8 5 8}$ \\
& $\begin{array}{l}\text { Moderado entre 21 i } \\
\text { 40\% de Elecciones }\end{array}$ & 1482 & 1489 & 2971 \\
& $\begin{array}{l}\text { Medio: entre 41 i } \\
\text { 60\% de Elecciones }\end{array}$ & 876 & 850 & 1726 \\
& $\begin{array}{l}\text { Alto: entre 61 i } \\
\text { 80\% de Elecciones }\end{array}$ & 300 & 220 & 520 \\
$\begin{array}{l}\text { Muy Alto: entre 81 i } \\
\text { 100\% de Elecciones }\end{array}$ & 30 & 29 & 59 \\
\hline Total & & 4023 & 4111 & 8134 \\
\hline
\end{tabular}

Fonte: Campo de pesquisa, organizado pela autora.

b) Exclusión o Antipatía: (n-0 de rechazos recibidos) Personas que reciben una actitud de marginación en el grupo. Es importante constatar la intensidad de dicho rechazo por ser un factor de aislamiento que puede responder a una actitud violencia del grupo ante una posible diversidad a la vez que puede ser un factor provocador deabsentismo o de respuesta violenta ante los demás. 308 C hicas y 444 chicos (752 en total) han recibido más de 16 rechaz os desu grupo lo quesin duda va a incidir en las actitudes y en el clima. Las causas mayoritariamente son complejas y no individuales.

Rev. Diálogo Educ., Curitiba, v. 9, n. 28, p. 417-439, set./ dez. 2009 
TABLA 4 - Rechazos Recibidos * Sexo

\begin{tabular}{|c|c|c|c|c|c|}
\hline & \multicolumn{2}{|c|}{ Sexo } & \multirow{2}{*}{$\begin{array}{l}\text { Total } \\
\text { Chica }\end{array}$} & \multirow[b]{2}{*}{$\%$} \\
\hline & & Chica & Chico & & \\
\hline \multirow{6}{*}{$\begin{array}{l}\text { Antipatía: } \\
\text { Rechazos } \\
\text { Recibidos }\end{array}$} & $\begin{array}{l}\text { Nula: } 0 \text { Rechazos } \\
\text { Recibidos }\end{array}$ & 487 & 479 & 966 & 11,13 \\
\hline & $\begin{array}{l}\text { Poca: entre } 1 \text { i } 5 \\
\text { Rechazos Recibidos }\end{array}$ & 2577 & 2214 & 4791 & 55,18 \\
\hline & $\begin{array}{l}\text { Media: entre } 6 \text { i } 10 \\
\text { Rechazos Recibidos }\end{array}$ & 688 & 925 & 1613 & 18,52 \\
\hline & $\begin{array}{l}\text { Alta: entre } 11 \text { i } 15 \\
\text { Rechazos Recibidos }\end{array}$ & 215 & 352 & 567 & 6,52 \\
\hline & $\begin{array}{l}\text { Muy Alta: entre } 16 \text { i } 20 \\
\text { Rechazos Recibidos }\end{array}$ & 50 & 117 & 167 & 1,92 \\
\hline & $\begin{array}{l}\text { Extrema: más de } 21 \\
\text { Rechazos Recibidos }\end{array}$ & 258 & 327 & 585 & 6,73 \\
\hline Total & & 4275 & 4414 & 8689 & \\
\hline
\end{tabular}

Fonte: Campo de pesquisa, organizado pela autora.

TABLA 5 - Índice de Antipatía * Sexo

(\% en relación al no ${ }^{0}$ de personas del grupo)

\begin{tabular}{|c|c|c|c|c|}
\hline & & \multicolumn{2}{|c|}{ Sexo } & \multirow{2}{*}{$\begin{array}{l}\text { Total } \\
\text { Chica }\end{array}$} \\
\hline & & Chica & Chico & \\
\hline \multirow[t]{5}{*}{$\begin{array}{l}\text { Índice de } \\
\text { Antipatía }\end{array}$} & $\begin{array}{l}\text { Bajo: entre } 0 \text { i } 20 \% \\
\text { de Rechazos }\end{array}$ & 2749 & 2380 & 5129 \\
\hline & $\begin{array}{l}\text { Poco: entre } 21 \text { i } 40 \% \\
\text { de Rechazos }\end{array}$ & 878 & 1042 & 1920 \\
\hline & $\begin{array}{l}\text { Medio: entre } 41 \text { i } \\
60 \% \text { de Rechazos }\end{array}$ & 294 & 482 & 776 \\
\hline & $\begin{array}{l}\text { Alto: entre } 61 \text { i } 80 \% \\
\text { de Redhazos }\end{array}$ & 81 & 170 & 251 \\
\hline & $\begin{array}{l}\text { Muy Alto: entre } 81 \text { i } \\
\text { 100\% de Redhazos }\end{array}$ & 21 & 37 & 58 \\
\hline Total & & 4023 & 4111 & 8134 \\
\hline
\end{tabular}

Fonte: Campo de pesquisa, organizado pela autora.

Rev. Diálogo Educ., Curitiba, v. 9, n. 28, p. 417-439, set./ dez. 2009 
c) Expansividad Positiva: Actitud abierta de la persona por querer participar en grupos amplios o con un número amplio de personas del grupo. 1745 C hicas y 2075 C hicos (3829 en total) muestran una reducida expansividad positiva

TABLA 6 - Expansividad Positiva: Elecciones Emitidas * Sexo

\begin{tabular}{|c|c|c|c|c|c|}
\hline & & \multicolumn{2}{|c|}{ Sexo } & \multirow{2}{*}{$\begin{array}{l}\text { Total } \\
\text { Chica }\end{array}$} & \multirow[b]{2}{*}{$\%$} \\
\hline & & Chica & Chico & & \\
\hline \multirow{6}{*}{$\begin{array}{l}\text { Expansividad } \\
\text { Positiva: } \\
\text { Elecciones } \\
\text { Emitidas }\end{array}$} & O Eleociones & 20 & 31 & 51 & 0,59 \\
\hline & $\begin{array}{l}\text { Poca: entre } 1 \text { i } 5 \\
\text { Eleociones }\end{array}$ & 1725 & 2044 & 3769 & 43,38 \\
\hline & $\begin{array}{l}\text { Media: entre } 6 \text { i } 10 \\
\text { Elecciones }\end{array}$ & 1516 & 1425 & 2941 & 33,89 \\
\hline & $\begin{array}{l}\text { Alta: entre } 11 \text { i } 15 \\
\text { Elecciones }\end{array}$ & 522 & 421 & 943 & 10,82 \\
\hline & $\begin{array}{l}\text { Muy Alta: entre } 16 \text { i } 20 \\
\text { Elecciones }\end{array}$ & 169 & 151 & 320 & 3,67 \\
\hline & $\begin{array}{l}\text { Extrema: más de } 21 \\
\text { Elecciones }\end{array}$ & 323 & 342 & 665 & 7,64 \\
\hline Total & & 4275 & 4414 & 8689 & \\
\hline
\end{tabular}

Fonte: Campo de pesquisa, organizado pela autora.

Rev. Diálogo Educ., Curitiba, v. 9, n. 28, p. 417-439, set./ dez. 2009 
TABLA 7 - Agrupación Índice de Expansividad Positiva* Sexo

\begin{tabular}{|c|c|c|c|c|}
\hline & & \multicolumn{2}{|c|}{ Sexo } & \multirow[t]{2}{*}{ Total } \\
\hline & & Chica & Chico & \\
\hline \multirow{5}{*}{$\begin{array}{l}\text { Índice de } \\
\text { Expansividad } \\
\text { Positiva }\end{array}$} & $\begin{array}{l}\text { Bajo: entre } 0 \text { i } 20 \% \\
\text { Elecciones }\end{array}$ & 1245 & 1491 & 2736 \\
\hline & $\begin{array}{l}\text { Poco: entre } 21 \text { i } 40 \% \\
\text { de Elecciones }\end{array}$ & 1680 & 1718 & 3398 \\
\hline & $\begin{array}{l}\text { Medio: entre } 41 \text { i } \\
60 \% \text { de Elecciones }\end{array}$ & 678 & 573 & 1251 \\
\hline & $\begin{array}{l}\text { Alto: entre } 61 \text { i } 80 \% \\
\text { de Elecciones }\end{array}$ & 272 & 204 & 476 \\
\hline & $\begin{array}{l}\text { Muy Alto: entre } 81 \text { i } \\
100 \% \text { de Elecciones }\end{array}$ & 133 & 111 & 244 \\
\hline Total & & 4008 & 4097 & 8105 \\
\hline
\end{tabular}

Fonte: Campo de pesquisa, organizado pela autora.

d) Expansividad N egativa: Actitud negativahacialas personas del grupo con las que no se comparten las actividades. D epende de quien hace el rechazo puede tener una gran influencia no sólo en las personas rechazadas sino en el grupo en general creando un clima de tensión. 553 chicas y 593 chicos (1146 en total) emiten más de 10 rechaz os lo que representa una cifra a considerar incluso para grupos de 25 personas.

Rev. Diálogo Educ., Curitiba, v. 9, n. 28, p. 417-439, set./ dez. 2009 
Una reinterpretación de la violencia en las escuelas

TABLA 8 - Expansividad Negativa: Rechazos Emitidos* Sexo

\begin{tabular}{|c|c|c|c|c|c|}
\hline & \multicolumn{2}{|c|}{ Sexo } & \multirow{2}{*}{$\begin{array}{l}\text { Total } \\
\text { Chica }\end{array}$} & \multirow[b]{2}{*}{$\%$} \\
\hline & & Chica & Chico & & \\
\hline \multirow{6}{*}{$\begin{array}{l}\text { Expansividad } \\
\text { Negativa: } \\
\text { Rechazos } \\
\text { Emitidos }\end{array}$} & $\begin{array}{l}0 \text { Rechazos } \\
\text { Emitidos }\end{array}$ & 192 & 214 & 406 & 4,69 \\
\hline & $\begin{array}{l}\text { Poca: entre } 1 \text { i } 5 \\
\text { Rechazos Emitidos }\end{array}$ & 2632 & 2087 & 5439 & 62,62 \\
\hline & $\begin{array}{l}\text { Media: entre } 6 \text { i } 10 \\
\text { Rechazos Emitidos }\end{array}$ & 898 & 800 & 1698 & 19,52 \\
\hline & $\begin{array}{l}\text { Alta: entre } 11 \text { i } 15 \\
\text { Rechazos Emitidos }\end{array}$ & 237 & 192 & 429 & 4,92 \\
\hline & $\begin{array}{l}\text { Muy Alta: entre } 16 \text { i } 20 \\
\text { Rechazos Emitidos }\end{array}$ & 48 & 75 & 123 & 1,41 \\
\hline & $\begin{array}{l}\text { Extrema: más de } 21 \\
\text { Rechazos Emitidos }\end{array}$ & 268 & 326 & 594 & 6,83 \\
\hline \multicolumn{2}{|l|}{ Total } & 4275 & 4414 & 8689 & \\
\hline
\end{tabular}

Fonte: Campo de pesquisa, organizado pela autora.

TABLA 9 - Índice de Expansividad Negativa* Sexo

\begin{tabular}{llcc|c}
\hline & & \multicolumn{2}{|c|}{ Sexo } & Total \\
\cline { 3 - 4 } & & Chica & Chico & \\
\hline $\begin{array}{l}\text { Índice de } \\
\text { Expansividad } \\
\text { Negativa }\end{array}$ & $\begin{array}{l}\text { Bajo: entre 0 i 20\% } \\
\text { de Rechazos }\end{array}$ & 2315 & 2542 & 4857 \\
& $\begin{array}{l}\text { Poco: entre 21 i 40\% } \\
\text { de Rechazos }\end{array}$ & 1249 & 1119 & 2368 \\
& $\begin{array}{l}\text { Medio: entre 41 i } \\
\text { 69\% de Rechazos } \\
\begin{array}{l}\text { Alto: entre 61 i 80\% } \\
\text { de Rechazos }\end{array}\end{array}$ & 301 & 270 & 571 \\
$\begin{array}{l}\text { Muy Alto: entre 81 i } \\
\text { 100\% de Rechazos }\end{array}$ & 48 & 119 & 214 \\
\hline Total & & 4008 & 4097 & 95 \\
\hline
\end{tabular}

Fonte: Campo de pesquisa, organizado pela autora.

Rev. Diálogo Educ., Curitiba, v. 9, n. 28, p. 417-439, set./ dez. 2009 
e) Índice de Autovaloración: conciencia de la persona del lugar que ocupa en el grupo. La baja autovaloración muestra poca confianza en las relaciones y la percepción alta una interpretación desajustada de las respuestas que se reciben y que son valoradas como positivas y reforzadoras de las relaciones que, en este caso, no son valoradas por el grupo.

TABLA 10 - Índice de Autovaloración anula el $0 *$ Sexo

\begin{tabular}{ll|cc|c|c}
\hline & \multicolumn{2}{|c|}{ Sexo } & Total & \\
\cline { 3 - 5 } & Chica & Chico & Chica & \% \\
\hline $\begin{array}{l}\text { Índice } \\
\text { Autovaloración } \\
\text { anula el 0 }\end{array}$ & $\begin{array}{l}\text { Valoración } \\
\text { imposible }\end{array}$ & 237 & 330 & 567 & 6,52 \\
& $\begin{array}{l}\text { Bajo menor de 70 } \\
\text { Adecuado entre }\end{array}$ & 1908 & 1904 & 3812 & 43,87 \\
& $\begin{array}{l}\text { 70 y 140 } \\
\text { A lto más de 140 }\end{array}$ & 862 & 1210 & 2478 & 28,54 \\
\hline Total & & & & & \\
\hline
\end{tabular}

Fonte: Campo de pesquisa, organizado pela autora.

\section{DISCUSIÓN / CONCLUSIONES}

El debate que se lleva a cabo, en el marco de la investigación, es de gran interés al incorporar las percepciones que tiene el profesorado, cómo se interceptan los datos de la dinámica grupal y sus posibles causas. Se analizan las ideas previas, las prácticas educativas, las necesidades y posibilidades y, por consiguiente, formas de intervenir. D estacamos como focos de interés:

- comentarios como: "siempre ha pasado", (en rechazos por imagen corporal, dificultades, cultura, trastornos) "reflejan los valores de la sociedad" (en relaciones de género, formas verbales de comunicarse, uso de móviles)... se ponen en entredicho porque, de alguna manera, cuestionan la eficacia el proceso educativo, las actuaciones en el marco del grupo y su percusión en la construcción de los valores relacionales;

- aparece el debate sobre la necesaria implicación educativa del profesorado que interviene en el grupo;

Rev. Diálogo Educ., Curitiba, v. 9, n. 28, p. 417-439, set./ dez. 2009 
- cuando se focaliza el análisis en el: rendimiento escolar el debate se orienta hacia modelo de escuela, inclusión, formas organizativas tipos de trabajo, éxito escolar.

En síntesis queda abierta le necesidad de profundizar el debate interno del centro educativo con el compromiso y liderzazo del profesorado asistente. Los ejes que se priorizan son:

- mejora del bienestar del alumnado en el grupo para posibilitar su sentimiento de pertenencia y buscar los motivos que pueden dificultarla;

- cambios en formas de distribuir el alumnado;

- implicación de diferentes áreas de aprendizaje para tratar los ejes detectados;

- mejora de la acción tutorial;

- interésparahallar respuestasquemejoren ladinámicarelacional;

- formas de trabajo cooperativo;

- clima de aula.

El hecho de que el profesorado sea agente de observación directa y de intervención es un factor importante para potenciar el cambio y lograr los objetivos propuestos.

El reto es colectivo para no dejar al alumnado excluido de los beneficios que ofrece el sistema educativo ya que además de no tener respuesta a un derecho y una necesidad se perpetúan las dificultades. Las acciones punitivas y excluyentes, no dan respuesta a situaciones complejas ni llegan a modificar las causas.

Por esto proponemos el trabajo en redes sociales como forma clave de acompañamiento al alumnado y sus familias y para dar fortaleza profesional en el debate y las acciones que se llevan a cabo.

\section{REFERENCIAS}

BRUNER, J. Actos de significado: más allá de la revolución cognitiva. Madrid: Alianza, 1998.

CO MELLAS, M. J. Las competencias para la acción tutorial. In: COMELLAS, M. J. (Coord.). Las competencias del profesorado para la acción tutorial. Barcelona: CissPraxis, 2002. p. 13-27.

Rev. Diálogo Educ., Curitiba, v. 9, n. 28, p. 417-439, set./ dez. 2009 
. ¿Cuándo el comportamiento de la infancia y la adolescencia se vuelve conflicto? Información, Barcelona, v. 27, p. 24-31, 2004.

Un observatorio abierto a la participación. Cuadernos de Pedagogía, Barcelona, n. 359, p. 79-84, 2006.

COMELLAS, M. J.; LOJO, M. En cavi de mirada per abordar i prevenir la violència a les escoles. Barcelona: O ctaedro, 2008.

DUBET, F. Les inégalités multipliées. Paris: L'aube, 2004.

GABERAN, P. La relation éducative. Ramonville: Eres, 2005.

HAMON, H. Tant qu'il y aura des élèves. Paris: Seuil, 2004.

MABIO N-BO NFILS, B. L'invention de la violence scolaire. France: Eres, 2005.

MARCHESI, A. Q ué será de nosotros los malos alumnos? Madrid: Alianza, 2005.

MEIREIU, P. La opción de educar. Barcelona: O ctaedro, 2001.

Referencias para un mundo sin referencias. Barcelona: Graó, 2004.

MORIN, E. O ù va le monde? Paris: L'Herme, 2007.

MUCCHIELLI, L. Violences et insécurité. Paris: La découverte, 2002.

PUGEAULT-CICCHELLI, C. ; CICCHELLI, V.; RAGI, T. Ce que nous savons des jeunes. Paris: PUF, 2004.

RAVEAUD, M. De l'enfant au citoyen. Paris: PUF, 2006.

ROJAS, M. L. Las semillas de la violencia. Madrid: Calpe, 2005.

SANTO SG UERRA, M. A. Hacia el futuro: riesgos y esperanzas. In: VVAA. Valores escolares y educación para la ciudadanía. Barcelona: Graó, 2002. p. 29-33.

TOCZEK, M-C.; MARTINOT, D. Le défi éducatif. Paris: Armand Colin, 2004. VAN HAECHK A. L'école des inégalités. Mons: Talus, 2001.

L'école à l'épreuve de la sociologie. Bruselas: De Boec, 2006.

VV.A A. Q u'avons nous fait du droit à l'éducation? Paris: L'Harmattan, 2007.

Recebido: 06/ 10/ 2008

Recibido: 10/ 06/ 2008

Aprovado: 12/ 12/ 2008

A probado: 12/ 12/ 2008

Revisado: 15/ 09/ 2009

Revisado: 09/ 15/ 2009

Rev. Diálogo Educ., Curitiba, v. 9, n. 28, p. 417-439, set./ dez. 2009 


\section{ANEXO 1- DATOS DE LA PARTICIPACIÓN POR CENTROS, PROFESORADO Y ALUMNADO}

Els centres han rebut una primera informació de l'alumnat seleccionat amb dades sociomètriques que han completat amb aportacions de dades pedagògiques.

A final de curs es lliurarà un informe a cada centre amb les dades dels grups en què s'ha treballat.

TABLA 1 - D atos de la participación por centros, profesorado y alumnado

\begin{tabular}{lccccc}
\hline $\begin{array}{l}\text { Comarca } \\
\text { (zona geográfica } \\
\text { que incluye } \\
\text { diferentes } \\
\text { poblaciones) }\end{array}$ & Poblaciones & Centro & Grupos & alumnado $^{\mathbf{1}}$ & Profesorado $^{\text {Promer }}$ \\
\hline Alt Empordà & 2 & 4 & 33 & 339 & 27 \\
Alt Penedès & 1 & 9 & 14 & 356 & 16 \\
Anoia & 9 & 11 & 6 & 82 & 28 \\
Alt Urgell & 1 & 2 & 18 & 263 & 13 \\
Barcelonès & 3 & 35 & 141 & 2621 & 140 \\
Baix Penedès & 1 & 5 & 13 & 357 & 25 \\
Garraf & 2 & 2 & - & - & 3 \\
Maresme & 2 & 2 & - & - & 3 \\
Montsià & 1 & 2 & 13 & 322 & 19 \\
Osona & 2 & 5 & 96 & 1331 & 96 \\
Urgell & 1 & 1 & 3 & 51 & 2 \\
Vallès Occidental & 3 & 11 & 73 & 1113 & 66 \\
Vallès Oriental & 3 & 7 & 12 & 300 & 16 \\
\hline Total & 31 & $\mathbf{1 0 1}$ & $\mathbf{4 2 2}$ & $\mathbf{7 1 3 5}$ & $\mathbf{4 5 4}$ \\
\hline
\end{tabular}

Fonte: Campo de pesquisa, organizado pela autora.

Aquest nombre és provisional i cal comptar que, de molts grups, hi ha informació de treball (que és la que consta) i de joc que no s’ha inclós com a població diferent perquè fan referència al mateix grup de població, malgrat sjobtinguin altres informacions.

Rev. Diálogo Educ., Curitiba, v. 9, n. 28, p. 417-439, set./ dez. 2009 